\title{
Link between obsessive-compulsive disorder and polymorphisms in HDAC genes
}

\author{
Ayse Dondu, ${ }^{1}$ Metin Caliskan, ${ }^{2}$ Seda Orenay-Boyacioglu ${ }^{2}$ iD \\ ${ }^{1}$ Department of Psychiatry, Aydın Goverment Hospital, Aydın, Turkey. ${ }^{2}$ Department of Medical Genetics, Faculty of Medicine, Aydın Adnan \\ Menderes University, Aydın, Turkey.
}

\begin{abstract}
Objective: Recently, epigenetic mechanisms related to histone modifications including histone deacetylation (HDAC) have been emphasized in psychiatric diseases. Few studies have investigated the relationship of HDAC gene variations to psychiatric diseases, but these gene variations have never been studied in obsessive-compulsive disorder (OCD). The present case-control study aimed to compare symptomatology with HDAC gene variations in patients with OCD.

Methods: Illumina next-generation sequencing of six HDAC genes (HDAC2,3,4,9,10,11) was performed on DNA samples isolated from 200 Turkish subjects recruited from routine clinical practice. Twenty-seven single nucleotide polymorphism (SNPs) in six HDAC genes were scanned with the LightSNiP method.

Results: New variants, all previously unreported in the literature, were identified in the HDAC4, $H D A C 10$, and $H D A C 11$ genes. When control and OCD patient groups were compared, a statistically significant difference was found in HDAC2 rs13212283, HDAC4 rs1063639, and HDAC10 rs1555048 in terms of genotype distribution ( $p<0.05$ ). In addition, in the OCD group, a statistically significant relationship was found between some obsessions/compulsions and HDAC2, HDAC3, and HDAC4 polymorphisms $(p<0.05)$.

Conclusions: Our study shows that the HDAC2, HDAC3, HDAC4, and HDAC10 genes may play a role in the pathogenesis of OCD.
\end{abstract}

Keywords: Obsessive-compulsive disorder; HDAC genes; histone modification; single nucleotide polymorphism; epigenetics

\section{Introduction}

Obsessive-compulsive disorder (OCD) is a psychiatric disorder where repeated obsessions or compulsions are observed continuously or periodically and clearly affect the person's functioning. ${ }^{1}$ The clinical presentation of OCD is heterogeneous. Genetic, epigenetic, environmental, psychological, social, and biochemical factors implicated in OCD etiology are thought to play a role in these differences in phenotype. ${ }^{2}$

A large body of evidence proves cortico-striatothalamo-corticalcircuitry dysfunction to the main pathophysiological feature of OCD., ${ }^{3,4}$ Structural brain abnormalities in the frontostriatal region have also been detected. ${ }^{5}$ The most consistent findings from functional imaging studies in OCD relate to abnormally increased activation of the lateral prefrontal cortex, including the orbitofrontal cortex and anterior cingulate. ${ }^{6}$ Furthermore, impairment is seen in several neuropsychological parameters, such as spatial working memory, spatial recognition, and cognitive processes related to motor initiation

Correspondence: Seda Orenay-Boyacioglu, Department of Medical Genetics, Faculty of Medicine, Aydın Adnan Menderes University, Efeler, 09010, Aydın, Turkey.

E-mail: sorenay@adu.edu.tr

Submitted Dec 21 2020, accepted Jul 13 2021, Epub Oct 292021 and execution, which suggests that OCD may have a relationship with neurodegenerative diseases (e.g., Alzheimer disease $[A D]) .^{7,8} \mathrm{~A}$ recent study by our group reported that lifetime OCD symptoms were significantly more common in $\mathrm{AD}$ patients compared to the control group. This information suggests that previously existing OCD symptoms may lead to a tendency to dementia in later ages in case of a real memory deficiency. ${ }^{9}$

Candidate gene studies have identified risk variables for OCD within serotonergic (HTR2A, 5HTTLPR, SLC6A4), glutamatergic (SLC1A1, DLGAP3, SAPAP3), and dopaminergic (SLC6A3, DRD4) genes, and genetic linkage studies in OCD have identified chromosomal regions (9p24, 3q27-28, 14q23-32 and sites on chromosomes 1, 6,7 , and 15) that contain genes for the disorder. Genomewide association studies (GWAS) of OCD have reported significant associations with the genes BTBD3, ASB13, RSPO4, DLGAP1, PTPRD, GRIK2, FAIM2, CDH2O, MEF2BNB, MEF2B, MEF2BNB-MEF2B, and RFXANK. ${ }^{10}$

In recent times, epigenetic mechanisms related to gene methylation, histone deacetylation (HDAC), and histone

How to cite this article: Dondu A, Caliskan M, Orenay-Boyacioglu S. Link between obsessive-compulsive disorder and polymorphisms in HDAC genes. Braz J Psychiatry. 2022;44:156-163. http://dx.doi. org/10.15 0/1516-4446-2020-1715 
acetylation (HAT) have been a focus in psychiatric disorders, and attempts have been made to create treatment strategies for these conditions. Different methylation profiles were found in the promoter regions of the MAOA, GABA, MOG, BDNF, LEPR, OXTR, SLC6A4, and $S L C 6 A 3$ genes in studies of DNA methylation comparing OCD patients and controls. ${ }^{11}$ The balance between HAT/ HDAC is thought to be related to neuron death, with diseases occurring when this balance is disrupted. ${ }^{12,13}$ Neuron cell death, causing amyloid precursor protein (APP) activation in AD and amyotrophic lateral sclerosis (ALS), was shown to be associated with HDAC levels in in vitro model studies. ${ }^{14}$ HAT has been characterized as an epigenetic mechanism involved in memory formation, and its relevance has been shown in both physiological and pathological conditions. Studies have shown HATrelated heterochromatin structure changes as long-term memories are formed. ${ }^{15,16}$ While several studies investigating the relationship of HDAC gene variations with psychotic and neurodevelopmental disorders have been published, these gene variations have not been studied for a potential role in OCD. Different studies have reported that HDAC4, HDAC3, HDAC10, and HDAC9 single nucleotide polymorphism (SNPs) are associated with schizophrenia, and a deletion on HDAC9 was reported in schizophrenia. ${ }^{17-21}$ Another intronic SNP of HDAC3 (rs2735188), reported by Anney et al., ${ }^{22}$ was associated with autism in genome screening of 1,558 families. According to animal studies, HAT has been implicated in mechanisms affecting memory processes in the hippocampus. ${ }^{16}$ In conclusion, variations in exonic and intronic regions of HDAC genes, playing roles in psychotic disorders, affect protein activity and thus cause suboptimal HAT in candidate genes, which is thought to increase OCD risk considerably. ${ }^{17-23}$ Within this context, we designed a casecontrol study to compare symptomatology and HDAC gene variations in patients with OCD.

\section{Methods}

The sample size in the study was determined using G-power. As there was no similar study related to the topic in the literature, we considered an effect size of $0.1,80 \%$ statistical power, a $95 \%$ confidence level and 0.05 type 1 error rate; the minimum sample size was calculated as 96 subjects. Our study ultimately included 160 patients and 40 controls.

A total of 160 patients attending the Aydın Government Hospital psychiatry clinic with a previous diagnosis of OCD according to the Structured Clinical Interview in Diagnostic and Statistical Manual of Mental Disorders IV were recruited. ${ }^{24,25}$ The Yale-Brown Obsessive Compulsive Scale (Y-BOCS) was applied to all patients to determine the severity of obsessive-compulsive (OC) symptoms. ${ }^{26}$ OC types were defined using the Y-BOCS symptom checklist. All patients were also administered the Hamilton Depression Rating Scale (HDRS) and Hamilton Anxiety Rating Scale (HARS) applied. The presence of OCDP was determined with the Structured Clinical Interview for DSM-IV Axis II Personality Disorders. As healthy controls, Aydın State Hospital personnel who had not been diagnosed with OCD and did not use any psychotropic drugs or other medications were recruited. Controls were matched with OCD cases in terms of age and sex. All subjects were screened for psychiatric and neurological diseases; the exclusion criteria were psychotic disorder, autism spectrum disorders, bipolar disorder, intellectual disability, substance use disorders, and any organic mental disorder diagnosis. Volunteers participating in the study completed a form created by the researchers to collect sociodemographic and clinical variables, age at disease onset and duration, and information on medical treatment. To prevent any impact on genetic data, patients with comorbid organic diseases or who were on any medication within the preceding 2 months were not included in the study.

\section{DNA isolation}

Peripheral blood was used to isolate DNA with the QIAamp DNA Blood Mini Kit (Qiagen, Hilden, Germany), following the manufacturer's instructions.

\section{DNA concentration and purity}

The concentration and purity of the isolated DNA samples were measured using a NanoDrop 1000 Spectrophotometer V3.7 (Thermo Scientific, Waltham, USA).

\section{Illumina sequencing}

DNA samples isolated from cases were sequenced for HDAC gene exons (HDAC2: 14 Exon, HDAC: 15 Exon, HDAC4: 27 Exon, HDAC9: 25 Exon, HDAC10: 20 Exon, and HDAC11: 9 Exon) and exon-intron junction points with next-generation sequencing (NGS) methods on the MiSeq Platform (Illumina, San Diego, USA). Raw data were analyzed according to the reference genome (GRCh37[h19]) in a web-based bioinformatics program (https://seq.genomize.com/). The rate at which total count numbers obtained at the end of the analysis encompassed the target regions was assessed as $100 \%$ using the Integrative Genomics Viewer (IGV) program. Variations identified according to $50 \mathrm{X}$ reading depth per allele (reference allele/alternative allele) were analyzed. Analysis of variations was completed in line with the Standards and Guidelines of the American College of Medical Genetics and Genomics (ACMG). Evaluation of variants in relevant databases (Ensembl, dbSNP, ClinVar, LOVD, UMD-MMR [Universal Mutation Database], HGMD ${ }^{\circledR}$ Professional 2017.3, InSiGHT, PubMed, MMR Variant Database) was made by taking the available information into account.

\section{Identification of variations}

The following criteria were applied in the evaluation of detected variations. Allele frequency must be $<5 \%$ in any of the following population databases: Exome Sequencing Project (ESP6500), 1000 Genomes Project (1000Genomes), and Exome Aggregation Consortium (ExAC). Exonic changes in all coding regions and 
exon-intron junction points ( $\pm 20 \mathrm{bp}$ ) have been taken into account. Variants in $3^{\prime}$ UTR and $5^{\prime}$ UTR were not evaluated. Polymorphisms were not indicated. Repeat expansions, copy number variants (CNV), large deletions and duplications, and mosaicism were not detected by this test.

\section{SNP selection}

In the study, gene polymorphisms in the HDAC intronic region, not previously studied for OCD patients but considered to be related to OCD, were chosen by screening the GWAS and ExAC databases and the literature related to other psychiatric diseases.

\section{Primer design}

The primers used in the study were designed by using the target sequence criteria stated below and by checking the primer suitability of the SNPs to be studied.

1. Length of target sequences must be between $60 \mathrm{bp}$ (including both upstream and downstream of the target SNP site) and $250 \mathrm{bp}$.

2. Only one SNP must be present in the target sequence.

3. For insertion/deletion procedures (In/Dels), the length of $\mathrm{In} / \mathrm{Del}$ must be less than $10 \mathrm{bp}$.

4. $\mathrm{G} / \mathrm{C}$ content of the target sequence must be $<65 \%$.

\section{LightSNiP method}

The SNPs found in the intron regions of the determined HDAC genes and associated with some psychiatric diseases were identified with real-time PCR (LightCycler 480 , Roche, Basel, Switzerland) using a SNiP panel from the manufacturer. Probes provided by the manufacturer identified single-base changes/SNPs, making polymorphism analysis possible. SNPs were analyzed by melting curve analysis at the end of amplification.

\section{Data analysis}

Data analysis was performed with LightCycler 480 software in Tm calling mode or with melting curve genotyping.

\section{Statistical analysis}

Statistical analyses were performed in SPSS for Windows 17.0. Differences between the patients and controls in terms of categorical variables, such as demographic and clinical data, were analyzed using chi-square $\left(\chi^{2}\right)$ tests, while continuous variables were analyzed with Student's $t$ test. Differences in allelic distribution of the 27 HDAC SNPs were also examined using $\chi^{2}$ test. The threshold of statistical significance was defined as 0.05 .

\section{Ethics statement}

This study was approved by the institutional ethics committee of Aydın Adnan Menderes University (\#2016/
1011). Before the study, all participants provided informed consent.

\section{Results}

Sociodemographic and clinical comparisons of the two groups are summarized in Table 1. There were significant differences between the OCD and control groups in terms of age, education level, and marital status $(p=0.003$, $p=0.001$, and $p=0.001$, respectively). There was no significant difference in the sex distribution of the groups $(p>0.05)$. As expected, the differences in tic history and HDRS and HARS scores between the OCD and control groups were also statistically significant $(p=0.001$, $p<0.001$, and $p<0.001$, respectively) (Table 1).

No defined variation was detected in any gene sequence analyses of $H D A C 2, H D A C 3$, and HDAC9 in the OCD and control groups.

For the HDAC4 gene, a novel (previously undefined) P855S (c.2563C $>$ T) heterozygote variant and p.T913M (c. $2738 \mathrm{C}>\mathrm{T})$ heterozygote variant were identified in two different OCD patients. In the control group, one case was identified to have a p.T913M (c.2738C > T) heterozygote variant.

For $H D A C 10$, one OCD patient had the undefined p.A334V (c.1001C $>$ T) heterozygote variant, one OCD patient had the undefined p.V544l (c.1630G $>A$ ) heterozygote/p.Q586R (c.1757A $>$ G) heterozygote/p.N612D $(c .1834 \mathrm{~A}>\mathrm{G})$ heterozygote triple variant, three OCD patients had the V429I (c.1285G $>$ A) heterozygote variant, and one OCD patient had the novel p.P366L (c.1097C > T) heterozygote variant identified. In the control group, one case had a novel p.V544I (c.1630G $>$ A) heterozygote/ p.Q586R (c.1757A $>$ G) heterozygote/p.N612D (c.183 $4 A>G$ ) homozygote triple variant, and one case had the p. $\mathrm{H} 543 \mathrm{~N}(\mathrm{c} .1627 \mathrm{C}>\mathrm{A})$ heterozygote variant identified.

For the HDAC11 gene, one OCD patient had the undefined p. $343 \mathrm{H}$ (c.1028C $>$ A) heterozygote variant identified.

The 25 polymorphism findings detected with the LightSNiP method are shown in Table 2. Comparisons of the control and OCD group identified statistically significant differences in HDAC2 rs13212283, HDAC4 rs1063639, and $H D A C 10$ rs 1555048 genotype distributions $(p=0.025$, $p=0.046$, and $p<0.001$, respectively). There were no statistically significant differences identified for genotype distributions of other SNPs between the groups ( $p>0.05)$, nor for allele frequency $(p>0.05)$.

Statistically significant differences were observed for some obsessions and compulsions in OCD patients with HDAC polymorphisms, which are shown in Table 3 $(p<0.05)$.

\section{Discussion}

This study is the first to assess the correlation between $O C D$ and $H D A C$ gene variations in Turkish patients. In our research, new variants of $H D A C 4, H D A C 10$, and $H D A C 11$ not previously reported in the literature were identified in cases, though without statistical significance. Comparisons of the OCD and control groups identified 
Table 1 Demographic and clinical comparison of groups

\begin{tabular}{|c|c|c|c|c|c|}
\hline Variable & OCD group $n(\%)$ & Control group n (\%) & df & $\chi^{2}$ & $p$-value \\
\hline \multicolumn{6}{|l|}{ Gender } \\
\hline Female & $132(82.50)$ & $25(62.50)$ & 1 & 0.245 & 0.160 \\
\hline Male & $28(17.50)$ & $15(37.50)$ & & & \\
\hline \multicolumn{6}{|l|}{ Marital status } \\
\hline Married & $71(44.37)$ & $24(60.00)$ & 3 & 4.949 & 0.001 \\
\hline Single & $72(45.00)$ & $14(35.00)$ & & & \\
\hline Divorced & $5(3.13)$ & $1(1.25)$ & & & \\
\hline Widowed & $12(7.50)$ & $1(1.25)$ & & & \\
\hline \multicolumn{6}{|l|}{ History of tics } \\
\hline Yes & $55(34.38)$ & $0(0.00)$ & 3 & 4.322 & 0.001 \\
\hline No & $105(65.62)$ & $0(0.00)$ & & & \\
\hline \multicolumn{6}{|l|}{ History of life triggers } \\
\hline Yes & $98(61.25)$ & & & & \\
\hline No & $62(38.75)$ & & & & \\
\hline \multicolumn{6}{|l|}{ OCD onset } \\
\hline Sudden & $56(35.00)$ & & & & \\
\hline Slow & $104(65.00)$ & & & & \\
\hline \multicolumn{6}{|l|}{ Obsessions } \\
\hline Aggression & $18(11.25)$ & & & & \\
\hline Contamination & $46(28.75)$ & & & & \\
\hline Symmetry & $31(19.38)$ & & & & \\
\hline Hoarding & 17 (10.62) & & & & \\
\hline Sexual & $5(3.13)$ & & & & \\
\hline Religious & $14(8.75)$ & & & & \\
\hline Somatic & $9(5.63)$ & & & & \\
\hline \multicolumn{6}{|l|}{ Compulsions } \\
\hline Repetition & $50(31.25)$ & & & & \\
\hline Cleaning & $12(7.50)$ & & & & \\
\hline Ritualistic & 17 (10.63) & & & & \\
\hline Counting & 37 (23.12) & & & & \\
\hline Ordering/arranging & $14(8.75)$ & & & & \\
\hline \multirow[t]{2}{*}{ Hoarding } & $17(10.62)$ & & & & \\
\hline & Mean \pm SD & Mean $\pm S D$ & $\mathrm{~F}$ & df & $\mathrm{p}$-value \\
\hline Age & $32.13 \pm 14.25$ & $32.55 \pm 8.13$ & 15.24 & 92 & $0.003^{\star *}$ \\
\hline Educational level (years) & $11.77 \pm 6.50$ & $10.60 \pm 10.77$ & 6.11 & 94 & $0.001^{\star \star *}$ \\
\hline HDRS & $16.51 \pm 9.18$ & $3.20 \pm 3.88$ & 25.29 & 86 & $<0.001^{\star \star *}$ \\
\hline HARS & $20.04 \pm 10.76$ & $4.03 \pm 4.67$ & 32.79 & 85 & $<0.001^{\star \star \star}$ \\
\hline OCD duration & $10.93 \pm 8.33$ & & & & \\
\hline Y-BOCS total & $22.54 \pm 9.17$ & & & & \\
\hline Y-BOCS obsession & $13.10 \pm 2.02$ & & & & \\
\hline Y-BOCS compulsion & $13.50 \pm 9.80$ & & & & \\
\hline
\end{tabular}

$\mathrm{df}=$ degrees of freedom; HARS = Hamilton Anxiety Rating Scale; HDRS = Hamilton Depression Rating Scale; OCD = obsessive-compulsive disorder; SD = standard deviation; Y-BOCS $=$ Yale-Brown Obsessive Compulsive Scale.

$* p<0.05, * * p<0.01, * * * p<0.001$.

statistically significant differences for the HDAC2 rs1321 2283, HDAC4 rs1063639, and HDAC10 rs1555048 SNPs. There were statistically significant differences observed for some obsessions and compulsions in OCD patients with HDAC polymorphisms. For sociodemographic features, significant differences were identified for marital status, age, education level, and tic history between OCD subjects.
Based on the assumption that OCD is a neurodevelopmental disease, it is expected that early-onset OCD may display higher heritability and be associated less with environmental stress factors. For late-onset OCD, some genetic and epigenetic mechanisms of stressful life events are believed to be effective in triggering the disease. The neurobiology of OCD is characterized by strong dysfunction in the serotonergic and dopaminergic 
Table 2 Genotype distribution of HDAC gene polymorphisms between groups

\begin{tabular}{|c|c|c|c|c|c|c|c|c|c|}
\hline \multirow{2}{*}{$\frac{\text { Gene/SNP marker }}{H D A C 2}$} & \multicolumn{3}{|c|}{ OCD group $(n=160) n(\%)$} & \multicolumn{3}{|c|}{ Control group $(n=40) n(\%)$} & \multirow[t]{2}{*}{$d f$} & \multirow[t]{2}{*}{$\chi^{2}$} & \multirow[t]{2}{*}{$p$-value } \\
\hline & & & & & & & & & \\
\hline Genotypes & AA & $A G$ & GG & AA & $A G$ & GG & & & \\
\hline rs13212283 & $40(25.00)$ & $40(25.00)$ & $80(50.00)$ & $15(37.50)$ & $5(12.50)$ & $20(50.00)$ & 2 & 6.310 & $0.025 *$ \\
\hline Genotypes & CC & CG & GG & $\mathrm{CC}$ & CG & GG & & & \\
\hline rs9488289 & $63(39.38)$ & $40(25.00)$ & $57(35.62)$ & $17(42.50)$ & $5(12.50)$ & $18(45.00)$ & 2 & 2.672 & 0.208 \\
\hline Genotypes & CC & CT & TT & CC & CT & TT & & & \\
\hline rs6568819 & $71(44.38)$ & $35(21.87)$ & $54(33.75)$ & $25(62.50)$ & $4(10.00)$ & $11(27.50)$ & 2 & 3.398 & 0.092 \\
\hline Genotypes & $\mathrm{CC}$ & CT & TT & $\mathrm{CC}$ & CT & TT & & & \\
\hline rs2499618 & $64(40.00)$ & $34(21.25)$ & $62(38.75)$ & $19(47.50)$ & $6(15.00)$ & $15(37.50)$ & 2 & 0.355 & 0.837 \\
\hline Genotypes & $A A$ & AT/G & $\mathrm{TT} / \mathrm{GG}$ & AA & AT/G & $\mathrm{TT} / \mathrm{GG}$ & & & \\
\hline rs13204445 & $60(37.50)$ & $35(21.87)$ & $65(38.13)$ & $17(42.50)$ & $6(15.00)$ & $17(42.50)$ & 2 & 0.110 & 0.946 \\
\hline Genotypes & CC & $\mathrm{CT}$ & TT & $\mathrm{CC}$ & CT & TT & & & \\
\hline rs10499080 & $83(51.87)$ & $46(28.75)$ & $31(19.38)$ & $30(75.00)$ & $6(15.00)$ & $4(10.00)$ & 2 & 3.818 & 0.100 \\
\hline \multicolumn{10}{|l|}{ HDAC3 } \\
\hline Genotypes & AA & $A G$ & GG & AA & $A G$ & GG & & & \\
\hline rs3844598 & $45(28.12)$ & $47(29.38)$ & $68(42.50)$ & $11(27.50)$ & $10(25.00)$ & $19(47.50)$ & 2 & 0.185 & 0.912 \\
\hline Genotypes & AA & $A G$ & GG & AA & AG & GG & & & \\
\hline rs 2530223 & $42(26.25)$ & $46(28.75)$ & $72(45.00)$ & $5(12.50)$ & $13(32.50)$ & $22(55.00)$ & 2 & 3.513 & 0.173 \\
\hline Genotypes & CC & CA & AA & $\mathrm{CC}$ & CA & AA & & & \\
\hline rs 142519 & $50(31.25)$ & $44(27.50)$ & $66(41.25)$ & $8(20.00)$ & $10(25.00)$ & $22(55.00)$ & 2 & 2.287 & 0.319 \\
\hline Genotypes & GG & GA & AA & GG & GA & AA & & & \\
\hline rs2735188 & $81(50.62)$ & $31(19.38)$ & $48(30.00)$ & $24(60.00)$ & $5(12.50)$ & $11(27.50)$ & 2 & 0.460 & 0.795 \\
\hline Genotypes & CC & CG & GG & CC & CG & GG & & & \\
\hline rs11742646 & 69 (43.12) & $35(21.88)$ & $56(35.00)$ & $21(52.50)$ & $5(12.50)$ & $14(35.00)$ & 2 & 0.515 & 0.773 \\
\hline Genotypes & CC & CG & GG & CC & CG & GG & & & \\
\hline rs 251041 & $42(26.24)$ & $59(36.88)$ & $59(36.88)$ & $9(22.50)$ & $14(35.00)$ & $17(42.50)$ & 2 & 0.287 & 0.866 \\
\hline \multicolumn{10}{|l|}{ HDAC4 } \\
\hline Genotypes & $\mathrm{CC}$ & CT & TT & $\mathrm{CC}$ & CT & TT & & & \\
\hline rs1063639 & 38 (23.75) & 37 (23.12) & $85(53.13)$ & $17(42.50)$ & $3(7.50)$ & $20(50.00)$ & 2 & 3.968 & $0.046 *$ \\
\hline Genotypes & GG & GA & AA & GG & GA & AA & & & \\
\hline rs3791480 & 33 (20.63) & $81(50.62)$ & $46(28.75)$ & $7(17.50)$ & $20(50.00)$ & $13(32.50)$ & 2 & 1.925 & 0.382 \\
\hline \multicolumn{10}{|l|}{$H D A C 9$} \\
\hline Genotypes & $\mathrm{CC}$ & CG & GG & $\mathrm{CC}$ & CG & GG & & & \\
\hline rs 1726596 & $86(53.75)$ & $33(20.62)$ & $41(25.63)$ & $26(65.00)$ & $4(10.00)$ & $10(25.00)$ & 2 & 1.582 & 0.453 \\
\hline Genotypes & AA & $A G$ & GG & AA & $A G$ & GG & & & \\
\hline rs12531908 & 39 (24.37) & $56(35.00)$ & 65 (40.63) & $11(27.50)$ & $12(30.00)$ & $17(42.50)$ & 2 & 2.282 & 0.320 \\
\hline Genotypes & $A A$ & $A G$ & GG & $A A$ & $A G$ & GG & & & \\
\hline rs7801162 & $56(35.00)$ & $43(26.87)$ & $61(38.13)$ & $16(40.00)$ & $7(17.50)$ & $17(42.50)$ & 2 & 0.945 & 0.623 \\
\hline Genotypes & TT & CT & CC & TT & CT & TT & & & \\
\hline rs2107595 & 71 (44.37) & $38(23.75)$ & $51(31.88)$ & $21(52.50)$ & $4(10.00)$ & $15(37.50)$ & 2 & 3.744 & 0.052 \\
\hline \multicolumn{10}{|l|}{ HDAC10 } \\
\hline Genotypes & TT & $\mathrm{TG} / \mathrm{C}$ & $\mathrm{GG} / \mathrm{CC}$ & TT & TG/C & GG/CC & & & \\
\hline rs 1555048 & $37(23.13)$ & $70(43.75)$ & $53(33.12)$ & $7(17.50)$ & $7(17.50)$ & $26(65.00)$ & 2 & 19.611 & $<0.001^{* * *}$ \\
\hline Genotypes & $\mathrm{CC}$ & CT & TT & CC & CT & TT & & & \\
\hline rs7290710 & $60(37.50)$ & $40(25.00)$ & $60(37.50)$ & $15(37.50)$ & $8(20.00)$ & $17(42.50)$ & 2 & 0.481 & 0.786 \\
\hline Genotypes & $\mathrm{AA}$ & $A G$ & GG & $A A$ & $A G$ & GG & & & \\
\hline rs 1076649 & $59(36.88)$ & $45(28.12)$ & $56(35.00)$ & $12(30.00)$ & 7 (17.50) & $21(52.50)$ & 2 & 4.658 & 0.097 \\
\hline Genotypes & AA & $A G$ & GG & AA & $A G$ & GG & & & \\
\hline rs74284 & $64(40.00)$ & $37(23.12)$ & $59(36.88)$ & $15(37.50)$ & $6(15.00)$ & $19(47.50)$ & 2 & 1.210 & 0.546 \\
\hline Genotypes & TT & TC & CC & TT & TC & CC & & & \\
\hline rs5771109 & $60(37.50)$ & $40(25.00)$ & $60(37.50)$ & $15(37.50)$ & $7(17.50)$ & $18(45.00)$ & 2 & 0.481 & 0.786 \\
\hline \multicolumn{10}{|l|}{$H D A C 11$} \\
\hline Genotypes & $\mathrm{CC}$ & CA & AA & $\mathrm{CC}$ & $\mathrm{CA}$ & AA & & & \\
\hline rs7634112 & $76(47.50)$ & $33(20.63)$ & $51(31.87)$ & $20(50.00)$ & $4(10.00)$ & $16(40.00)$ & 2 & 2.414 & 0.299 \\
\hline Genotypes & TT & TG & GG & TT & TG & GG & & & \\
\hline rs17038236 & $88(55.00)$ & 31 (19.37) & $41(25.63)$ & $24(60.00)$ & $5(12.50)$ & $11(27.50)$ & 2 & 1.717 & 0.424 \\
\hline
\end{tabular}

$\mathrm{df}=$ degrees of freedom; HDAC = histone deacetylation; $\mathrm{OCD}=$ obsessive-compulsive disorder; $\mathrm{SNP}=$ single nucleotide polymorphism.

$* p<0.05, * * p<0.01, * * * p<0.001$.

systems. Accordingly, family, twin, segregation, and linkage studies of OCD have demonstrated roles of genes related to the serotonergic and dopaminergic system. ${ }^{27}$ Recently, the focus has shifted to epigenetic mechanisms related to modifications including gene methylation, HDAC, and HAT in psychiatric diseases, and attempts have been made to create treatment strategies that address these mechanisms. ${ }^{17-23}$ Within 


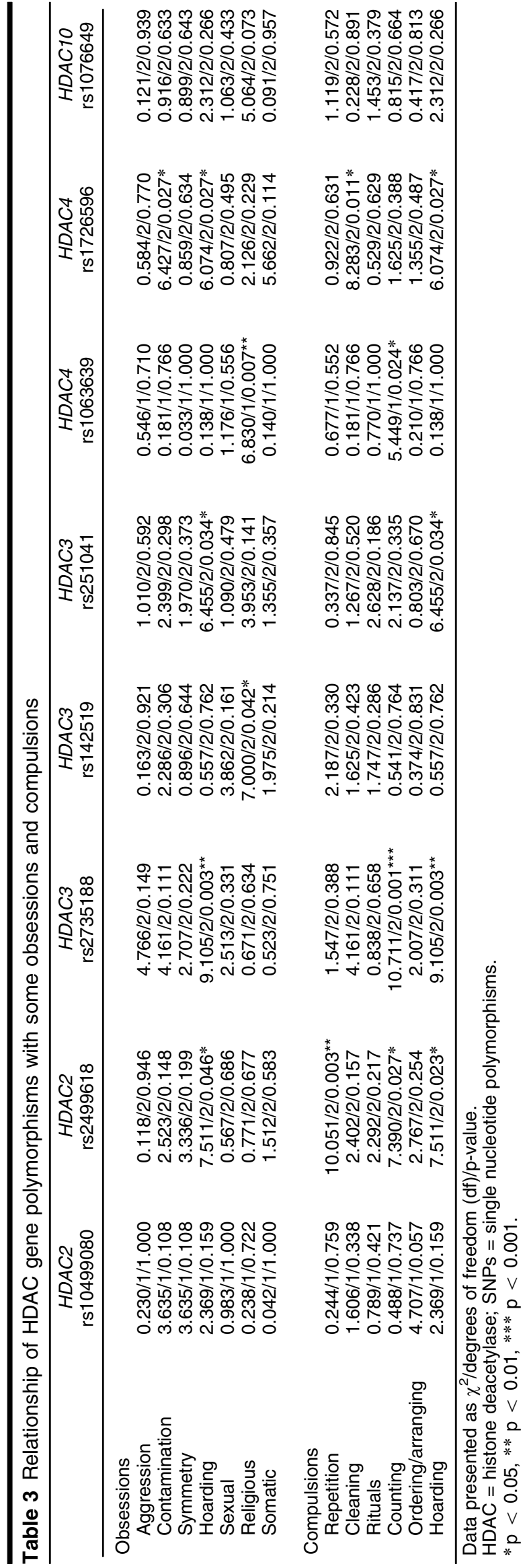

this context, the present study researched the correlation between HDAC gene variations and OCD, as these variations may be an epigenetic mechanism that regulates the expression of candidate genes involved in OCD genetics.

While studies have investigated the correlation of HDAC gene variations with some psychotic diseases, these variations have not been studied for OCD. A casecontrol study in the Korean population reported an SNP in HDAC4 associated with schizophrenia, and a deletion in HDAC9 in schizophrenia. ${ }^{18}$ Additionally, HDAC4 mutations were identified in brachydactyly-mental retardation syndrome involving autistic symptoms. ${ }^{19}$ In a thesis, Wedenoja ${ }^{20}$ studied SNPs of $2 q 33.1-2 q 37.3$, 4q13.1-4q26, and 5q31.1-5q33.3 chromosome regions connected with schizophrenia for all 1,511 intragenic and adjacent tagSNPs of candidate genes and chose 104 genes based on the International HapMap Project CEPH genotype data. Among the chosen 104 candidate genes, the author included HDAC3 (rs3844598, rs117 42646, rs251041) and HDAC4 (rs3791480) SNP regions and identified a significant correlation between these SNP regions with schizophrenia. The highest significance was identified for HDAC3 rs3844598. ${ }^{20}$ Our study examined the same SNPs; however, statistically significant results were not obtained. The reason for this is thought to be the genetic basis of different disorders, which may not overlap. An SNP study by Kim et al. ${ }^{17}$ in a Korean population of patients with schizophrenia researched HDAC3 (rs2530223), HDAC4 (rs1063639), and HDAC10 (rs1555048) SNPs and concluded that the HDAC3 and HDAC4 genes may play an important role in schizophrenia pathophysiology. In our study, parallel to the study by Kim et al., ${ }^{17}$ the HDAC4 rs1063639 SNP was significant in OCD patients, and this SNP may be involved in OCD pathogenesis. Kebir et al. ${ }^{21}$ found correlations for HDAC10 locus-localized rs1076649, rs5771109, and rs742184, HDAC9 gene-localized rs1726596, rs12531908, and rs7801662, HDAC3 rs14251, and HDAC11 rs7634112 and rs17038236 SNPs with schizophrenia in an exome sequencing study related to rare mutations of HDAC genes in 1,134 schizophrenia cases. ${ }^{21}$ Anney et al. ${ }^{22}$ reported a correlation with autism for another intronic SNP of HDAC3 (rs2735188) during genome screening including 1,558 families. In our study, polymorphisms previously reported by Kebir et al. ${ }^{21}$ and Anney et al. $^{22}$ were screened, but no correlation was found for OCD. The reason for this is thought to be the low number of OCD subjects in the current study, and, as mentioned above, the fact that the genetic basis of different disorders may not overlap. Chen et al. ${ }^{28}$ studied 1,430 schizophrenic and 2,562 control patients in the Chinese population and researched three different HDAC2 SNP regions (rs13212283, rs6568819, and rs9488289), and did not obtain any significant results. In our study, a connection was found for the rs13212283 polymorphism of the HDAC2 gene with OCD. Again, this difference compared to the results of Chen et al. ${ }^{28}$ is thought to be due to ethnic background and to the fact that genetic bases of different disorders may not overlap. Han et al. ${ }^{29}$ chose HDAC2 (rs10499080, rs6568819, 
rs2499618, and rs13204445) and HDAC3 (rs11741808, rs2530223) SNPs in trio analysis of 208 families with schizophrenia and did not identify a correlation between schizophrenia and these SNP regions. These results are consistent with ours. In our study, all exon and exon-intron junction regions for $H D A C 2, H D A C 3, H D A C 4, H D A C 9$, $H D A C 10$, and $H D A C 11$ were screened and novel variants were identified for HDAC4, HDAC10, and HDAC11. With the LightSNiP method, there were statistically significant differences identified for the HDAC2 rs13212283, HDAC4 rs1063639, and HDAC10 rs1555048 SNPs.

In addition, a statistically significant relationship was found between increased frequency of repetitive ritual obsessions, hoarding obsessions/compulsions, counting compulsions, and HDAC2, HDAC3, and HDAC4 polymorphisms in the OCD group, suggesting that these polymorphisms may represent a distinct clinical and psychobiological profile in OCD. In OCD, ritual-repetition obsessions are especially thought to be related to problems with the patient's memory, and studies have focused on this topic in the literature. ${ }^{23}$ It has been reported that symptoms such as symmetry obsession, ordering, ritual repetition, and counting compulsion are observed in patients showing hoarding symptoms more often than in those who do not exhibit hoarding. ${ }^{30}$ Similar inferences were made by Pertusa et al., ${ }^{31}$ who proposed classifying hoarding as a different form of OC symptoms, unlike symmetry obsession, ordering, ritual repetition, and counting compulsion. Hoarding as a symptom is also linked to Tourette's syndrome, chronic motor tic disorder, and OCPD. ${ }^{31}$ Therefore, the fact that these findings may stem from a mutual underlying root cannot be neglected. Hoarding is separate from OCD and classified as a separate disease group in the DSM-V. On the other hand, it may be suggested that the fundamental nature of hoarding can bring about secondary behavioral disorders, as it may only be natural to expect one to count or arrange the collected items. ${ }^{30,31}$

On comparison of the presence and absence of obsessions and compulsions individually, the fact that these polymorphisms are observed more frequently in the absence of certain obsessions/compulsions suggests that these polymorphisms may have a protective effect on symptom onset in OCD patients. From this aspect, our study shows that variants identified by both the NGS and LightSNiP methods on the HDAC2, HDAC3, HDAC4, and $H D A C 10$ genes may play a role in OCD pathogenesis and genetic etiology. This is the first study to assess the correlation between OCD and HDAC gene variations. However, further research with larger samples is needed to identify more significant variants and whether the association of these variants with OCD prevails.

\section{Acknowledgements}

This research was funded by the Scientific and Technological Research Council of Turkey (TUBITAK), grant number 317 S076.

\section{Disclosure}

The authors report no conflicts of interest.

\section{References}

1 Kiejna A, Rymaszewska J, Kantorska-Janiec M, Tokarski W. [Epidemiology of obsessive-compulsive disorder]. Psychiatr Pol. 2002; 36:539-48.

2 Nissen JB, Hansen CS, Starnawska A, Mattheisen M, Borglum AD, Buttenschøn $\mathrm{HN}$, et al. DNA methylation at the neonatal state and at the time of diagnosis: preliminary support for an association with the estrogen receptor 1 , gamma-aminobutyric acid $B$ receptor 1 , and myelin oligodendrocyte glycoprotein in female adolescent patients with OCD. Front Psychiatry. 2016;7:35.

3 Pompanin S, Perini G, Toffanin T, Gnoato F, Cecchin D, Manara R, et al. Late-onset $O C D$ as presenting manifestation of semantic dementia. Gen Hosp Psychiatry. 2012;34:102-e1-4.

4 Welch JM, Lu J, Rodriguiz RM, Trotta NC, Peca J, Ding JD, et al. Cortico-striatal synaptic defects and OCD-like behaviours in Sapap3mutant mice. Nature. 2007;448:894-900.

5 Purcell R, Maruff P, Kyrios M, Pantelis C. Cognitive deficits in obsessive-compulsive disorder on tests of frontal-striatal function. Biol Psychiatry. 1998;43:348-57.

6 Welter ML, Burbaud P, Fernandez-Vidal S, Bardinet E, Coste J, Piallat B, et al. Basal ganglia dysfunction in OCD: subthalamic neuronal activity correlates with symptoms severity and predicts highfrequency stimulation efficacy. Transl Psychiatry. 2011;1:e5.

7 McGrath LM, Yu D, Marshall C, Davis LK, Thiruvahindrapuram B, Li $\mathrm{B}$, et al. Copy number variation in obsessive-compulsive disorder and tourette syndrome: a cross-disorder study. J Am Acad Child Adolesc Psychiatry. 2014;53:910-9.

8 Khiari HM, Achouri A, Ali NB, Cherif A, Batti H, Messaoud T, et al. Obsessive-compulsive disorder: a new risk factor for Alzheimer disease? Neurol Sci. 2011;32:959-62.

9 Dondu A, Sevincok L, Akyol A, Tataroglu C. Is obsessive-compulsive symptomatology a risk factor for Alzheimer-type dementia? Psychiatry Res. 2015;225:381-6.

10 Purty A, Nestadt G, Samuels JF, Viswanath B. Genetics of obsessive-compulsive disorder. Indian J Psychiatry. 2019;61:S37-42.

11 Bellia F, Vismara M, Annunzi E, Cifani C, Benatti B, Dell'Osso B, et al. Genetic and epigenetic architecture of obsessive-compulsive disorder: in search of possible diagnostic and prognostic biomarkers. J Psychiatr Res. 2021;137:554-71.

12 Dietz KC, Casaccia P. HDAC inhibitors and neurodegeneration: at the edge between protection and damage. Pharmacol Res. 2010;62:11-7.

13 Saha RN, Pahan K. HATs and HDACs in neurodegeneration: a tale of disconcerted acetylation homeostasis. Cell Death Differ. 2006;13: 539-50.

14 Harrison IF, Dexter DT. Epigenetic targeting of histonedeacetylase: therapeutic potential in Parkinson's disease? Pharmacol Ther. 2013; 140:34-52.

15 Levenson JM, O'Riordan K J, Brown KD, Trinh MA, Molfese DL, Sweatt JD. Regulation of histone acetylation during memory formation in the hippocampus. J Biol Chem. 2004;279:40545-59.

16 Peixoto $\mathrm{L}$, Abel $\mathrm{T}$. The role of histone acetylation in memory formation and cognitive impairments. Neuropsychopharmacology. 2013; 38:62-76.

17 Kim T, Park JK, Kim HJ, Chung JH, Kim JW. Association of histone deacetylase genes with schizophrenia in Korean population. Psychiatry Res. 2010;178:266-9.

18 Lang B, Alrahbeni TM, St Clair D, Blackwood DH, International Schizophrenia Consortium; McCaig CD, et al. HDAC9 is implicated in schizophrenia and expressed specifically in post-mitotic neurons but not in adult neural stem cells. Am J Stem Cells. 2012;1:31-41.

19 Williams SR, Aldred M A, Der Kaloustian VM, Halal F, Gowans G, McLeod DR, et al. Haploinsufficiency of HDAC4 causes brachydactyly mental retardation syndrome, with brachydactyly type $E$, developmental delays, and behavioral problems. Am J Hum Genet. 2010;87:219-28.

20 Wedenoja J. Molecular genetics of schizophrenia and related intermediate phenotypes in a founder population [dissertation]. University of Helsinki: Helsinki; 2010.

21 Kebir O, Chaumette B, Fatjó-Vilas M, Ambalavanan A, Ramoz N, Xiong $L$, et al. Family-based association study of common variants, rare mutation study and epistatic interaction detection in HDAC genes in schizophrenia. Schizophr Res. 2014;160:97-103. 
22 Anney R, Klei L, Pinto D, Regina Regan, Judith Conroy, Tiago R Magalhaes, et al. A genome-wide scan for common alleles affecting risk for autism. Hum Mol Genet. 2010;19:4072-82.

23 Tolin DF, Abramowitz JS, Brigidi BD, Amir N, Street GP, Foa EB. Memory and memory confidence in obsessive-compulsive disorder. Behav Res Ther. 2001;39:913-27.

24 American Psychiatric Association. Handbook on the Definition and Classification of Diseases in Psychiatry, fourth revised edition (DSMIV-TR). (Translation ed. E Köroğlu) Ankara: Physicians Publishing Union; 2000.

25 First MB, Spitzer RL, Gibbon M, Williams JB. User's guide for the Structured clinical interview for DSM-IV axis I disorders SCID-I: clinician version. Washington: American Psychiatric Publishing; 1997.

26 Goodman WK, Price LH, Rasmussen SA, Mazure C, Fleischmann RL, Hill CL, et al. The Yale-Brown obsessive compulsive scale: I. Development, use, and reliability. Arch Gen Psychiatry. 1989;46:1006-11.
27 Yoo SW, Kim SJ, Kim CH. Association between obessive-compulsive disorder and dopamine transporter gene polymorphism. Psychiatry Investig. 2006;3:72-7.

28 Chen G, Guan F, Lin H, Li L, Fu D. Genetic analysis of common variants in the HDAC2 gene with schizophrenia susceptibility in Han Chinese. J Hum Genet. 2015;60:479-84.

29 Han H, Yu Y, Shi J, Yao Y, Li W, Kong N, et al. Associations of histone deacetylase-2 and histone deacetylase-3 genes with schizophrenia in a Chinese population. Asia Pac Psychiatry. 2013; 5:11-6.

30 Fontenelle LF, Mendlowicz MV, Soares ID, Versiani M. Patients with obsessive-compulsive disorder and hoarding symptoms: a distinctive clinical subtype?. Compr Psychiatry. 2004;45:375-83.

31 Pertusa A, Frost RO, Fullana MA, Samuels J, Steketee G, Tolin D, et al. Refining the diagnostic boundaries of compulsive hoarding: a critical review. Clin Psychol Rev. 2010;30:371-86. 